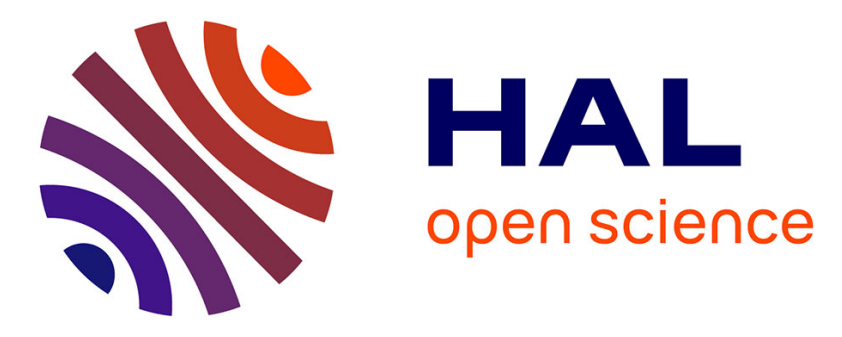

\title{
Exploring charge density analysis in crystals at high pressure: data collection, data analysis and advanced modelling
}

Nicola Casati, Alessandro Genoni, Benjamin Meyer, Anna Krawczuk, Piero Macchi

\section{To cite this version:}

Nicola Casati, Alessandro Genoni, Benjamin Meyer, Anna Krawczuk, Piero Macchi. Exploring charge density analysis in crystals at high pressure: data collection, data analysis and advanced modelling. Acta Crystallographica Section B : Structural Science, Crystal Engineering and Materials [2014-...], 2017, 73 (4), pp.584-597. 10.1107/S2052520617008356 . hal-02196486

\section{HAL Id: hal-02196486 \\ https://hal.univ-lorraine.fr/hal-02196486}

Submitted on 27 May 2020

HAL is a multi-disciplinary open access archive for the deposit and dissemination of scientific research documents, whether they are published or not. The documents may come from teaching and research institutions in France or abroad, or from public or private research centers.
L'archive ouverte pluridisciplinaire HAL, est destinée au dépôt et à la diffusion de documents scientifiques de niveau recherche, publiés ou non, émanant des établissements d'enseignement et de recherche français ou étrangers, des laboratoires publics ou privés. 


\section{Exploring charge density analysis in crystals at high pressure: data collection, data analysis and advanced modelling}

\section{Nicola Casati, Alessandro Genoni, Benjamin Meyer, Anna Krawczuk and Piero Macchi}

Acta Cryst. (2017). B73, 584-597

\section{IUCr Journals CRYSTALLOGRAPHY JOURNALS ONLINE}

Copyright (C) International Union of Crystallography

Author(s) of this paper may load this reprint on their own web site or institutional repository provided that this cover page is retained. Republication of this article or its storage in electronic databases other than as specified above is not permitted without prior permission in writing from the IUCr.

For further information see http://journals.iucr.org/services/authorrights.html 


\section{Philip Coppens tribute}

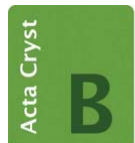

STRUCTURAL SCIENCE CRYSTAL ENGINEERING MATERIALS

ISSN 2052-5206

Received 6 March 2017

Accepted 6 June 2017

Edited by C. Lecomte, Université de Lorraine, France

Keywords: X-ray-constrained wavefunctions; multipolar model; high pressure diffraction; charge density.

Supporting information: this article has supporting information at journals.iucr.org/b

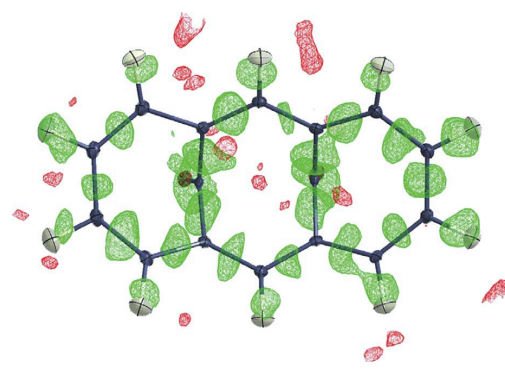

(C) 2017 International Union of Crystallography

\section{Exploring charge density analysis in crystals at high pressure: data collection, data analysis and advanced modelling}

\author{
Nicola Casati, ${ }^{a}$ Alessandro Genoni, ${ }^{\text {b,c }}$ Benjamin Meyer, ${ }^{\text {b,c }}$ Anna Krawczuk ${ }^{d}$ and \\ Piero Macchi ${ }^{\mathrm{e}}$ *
}

The possibility to determine electron-density distribution in crystals has been an enormous breakthrough, stimulated by a favourable combination of equipment for X-ray and neutron diffraction at low temperature, by the development of simplified, though accurate, electron-density models refined from the experimental data and by the progress in charge density analysis often in combination with theoretical work. Many years after the first successful charge density determination and analysis, scientists face new challenges, for example: (i) determination of the finer details of the electron-density distribution in the atomic cores, (ii) simultaneous refinement of electron charge and spin density or (iii) measuring crystals under perturbation. In this context, the possibility of obtaining experimental charge density at high pressure has recently been demonstrated [Casati et al. (2016). Nat. Commun. 7, 10901]. This paper reports on the necessities and pitfalls of this new challenge, focusing on the species syn1,6:8,13-biscarbonyl[14]annulene. The experimental requirements, the expected data quality and data corrections are discussed in detail, including warnings about possible shortcomings. At the same time, new modelling techniques are proposed, which could enable specific information to be extracted, from the limited and less accurate observations, like the degree of localization of double bonds, which is fundamental to the scientific case under examination.

\section{Introduction}

Since the pioneering studies by Coppens (1967), Stewart (1970), Hirshfeld (1971) and many others, the field of experimental charge density analysis has grown enormously and reached a status of maturity, as certified by Coppens (2005). Although experiments to map the charge density cannot be considered 'routine', efforts necessary nowadays to obtain a standard electron distribution from an X-ray diffraction experiment on single crystals are less than those necessary in the 1960s for 'just' a crystal structure determination. In the charge density language, standard means highquality single-crystal X-ray diffraction measurements, with a sufficient suitability factor (Stevens \& Coppens, 1976), at liquid nitrogen temperature (and ambient pressure), collecting with sufficient redundancy a full sphere of data up to a resolution of at least $d=0.5 \AA$ (i.e. $\sin \theta / \lambda=1.0 \AA^{-1}$ ) for organic/organometallic crystals with analytical correction of absorption effects, and a sufficiently high intensity/noise ratio. A higher resolution is necessary for metals, alloys and inorganic systems (Pillet et al., 2001; Schmøkel et al., 2013). Nowadays, these kinds of measurements can be carried out in 
laboratories (in $c a$ 24-72 h) or at synchrotron stations (within one shift of $8 \mathrm{~h}$ ). This improvement is due to modern detecting technologies, the more intense sources available on laboratory equipment, or the superior brilliance of synchrotron light, reliable cooling devices and progress in data correction and modelling.

This achieved maturity is evidence of progress that fostered more advanced investigations. For example, accurate studies at extremely high resolution $\left(d<0.35 \AA\right.$, $\left.\sin \theta / \lambda>1.4 \AA^{-1}\right)$ enable more accurate modelling of the core deformations and the radial density (Fischer et al., 2011). Combined X-ray and polarized neutron diffraction experiments allowed the determination of charge and spin density simultaneously (Deutsch et al., 2014), or combined elastic and inelastic scattering experiments enabled the partial reconstruction of the firstorder reduced density matrix (Gillet et al., 2001). Moreover, comprehensive data collections of chemically similar species may favour a multivariate analysis of the electron-density descriptors, similar to the widely adopted structure correlation method proposed by Bürgi \& Dunitz (1994). Finally, measurements of systems under some special perturbation, such as an electric field (Elsässer \& Woerner, 2012; Fertey et al., 2013), photo-excitation (Legrand et al., 2005), high temperature or pressure (Casati et al., 2016), return electrondensity distributions corresponding to the electronic states that significantly deviate from the gas-phase ground state of a molecule, therefore containing an enormous source of information.

Having this in mind, some of us started a long-term project with the purpose of obtaining accurate information on electron distributions from molecular crystals under high-pressure conditions. The aim is to observe in detail the changes occurring to the electronic structure of molecules embedded

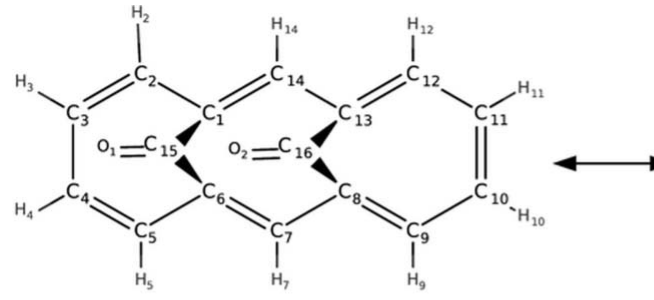

$\boldsymbol{A}$

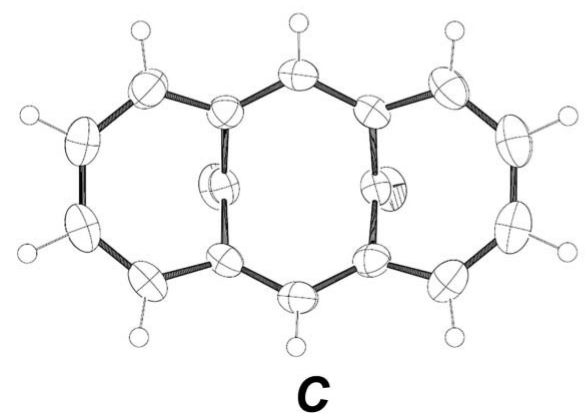

Figure 1

Top: Resonance structures $A$ and $B$ for the syn-1,6:8,13-biscarbonyl[14]annulene molecule. Bottom: Molecular geometry at ambient pressure $(C)$ and at $7.7 \mathrm{GPa}(D)$ (atomic displacement parameters are drawn at the $50 \%$ probability level). in volumes that are much smaller than those typical of crystals in thermodynamic equilibrium at ambient pressure. Upon compression, the molecules may modify their electronic states, and even react inside a crystal forming adducts (and eventually polymers) that are otherwise unstable.

So far, only a few studies have reported on attempts to determine electron densities at high pressure, for example, trying a multipolar model (Fabbiani et al., 2011; Macchi \& Casati, 2011) or a maximum entropy method (MEM; Yamanaka et al., 2009; Tse et al., 2006) refinement.

In a recent paper, some of us reported a complete multipolar model refined against data collected at high pressure, see Casati et al. (2016). The molecule under investigation is syn1,6:8,13-biscarbonyl[14] annulene, hereinafter BCA, see Fig. 1. This compound was the subject of an accurate electron-density study by Destro \& Merati (1995) that attracted interest because of the unusual finding of a bond path linking the two $\mathrm{C}$ atoms of the bridging carbonyl group, which inaugurated a series of similar observations in many other molecules.

The observed and calculated high-pressure evolution of BCA is extremely interesting (Casati et al., 2016), because the almost $C_{2 v}$ molecular symmetry featured under ambient conditions evolves towards an asymmetric structure, which localizes the double bonds of only one of the two resonant configurations of the ideal aromatic system (see Fig. 1). It is noteworthy, because BCA crystallizes in $P 2_{1} / n$ under ambient conditions, that the molecule necessarily sits on a general position and the gradual changes to its geometry are not accompanied by a solid-state phase transition.

In this paper, we report a detailed analysis of the collected data, of the derived multipolar models and of the X-rayconstrained wavefunctions reported in Casati et al. (2016). Moreover, we add an additional analysis based on the use of extremely localized molecular orbitals (ELMOs).

This experiment and data analysis enabled us to draw some general conclusions about the necessities and pitfalls of accurate electron-density determination at high pressure, a field that could develop in the near future, especially thanks to progress in the available equipment.

\section{Necessities and pitfalls of high-pressure high-resolution data collection}

As introduced above, a standard electron-density determination requires: (i) a suitable species; (ii) high-quality crystals that could guarantee sufficient diffraction at high resolution; (iii) $100 \%$ completeness of the data collected at a resolution of at least $d=0.5 \AA$ 
Table 1

The expected data coverage for data collection in a DAC with an opening of $80^{\circ}$ for some selected Laue classes.

The simulation was performed for the following unit cell: $a=b=c=10 \AA$ and $\alpha=\beta=\gamma=90^{\circ}$.

\begin{tabular}{llllll}
\hline $\begin{array}{l}\text { Resolution } \\
(d)(\AA)\end{array}$ & $\begin{array}{l}\text { Cell aperture } \\
\left({ }^{\circ}\right)\end{array}$ & Laue class & $\begin{array}{l}\lambda=0.71 \AA) \\
(\%)\end{array}$ & $\begin{array}{l}\lambda=0.56 \AA) \\
(\%)\end{array}$ & $\begin{array}{l}\lambda=0.30 \AA \\
(\%)\end{array}$ \\
\hline 0.83 & \multirow{2}{*}{80} & $\overline{1}$ & 37 & 40 & 53 \\
& & $2 / m$ & 39 & 44 & 54 \\
& & $m m m$ & 42 & 48 & 56 \\
& & $4 / m m m$ & 69 & 76 & 86 \\
& & $m \overline{3} m$ & 85 & 93 & 98 \\
0.5 & & & & \\
& & & & & \\
& & & & & \\
& & $2 / m$ & 14 & 24 & 45 \\
& & $m m m$ & 18 & 31 & 46 \\
& & $4 / m m m$ & 32 & 53 & 78 \\
& & $m \overline{3} m$ & 42 & 68 & 94 \\
\hline
\end{tabular}

( $\sin \theta / \lambda=1.0 \AA^{-1}$ ); (iv) accurate and precise measurement of the X-ray scattered intensities; (v) accurate data correction, especially for the absorption effects; (vi) sufficient low temperature ( $T \leq 120 \mathrm{~K}$ for organic/organometallic species) for a better deconvolution of the static electron-density from the displacement of atomic nuclei.

In order to collect single-crystal X-ray diffraction data at high pressure (at least above $0.1 \mathrm{GPa}$ ), a diamond anvil cell (DAC) is normally used (Boldyreva, 2008; Katrusiak, 2008). This equipment inherently hampers some of the abovementioned fundamental requirements for a standard electrondensity determination (see Fig. 2). In particular, the reduced aperture of a DAC (typically $<90^{\circ}$ ) hampers the possibility of collecting a full sphere of data, even to a low resolution. In fact, depending on the symmetry of the crystal system, it is impossible to measure some unique reflections. For a resolution of $d=0.83 \AA$, using conventional Mo $K \alpha$ radiation, less than $40 \%$ of the reflections of a crystal of Laue class $\overline{1}$ are measurable and, anyway, no more than $85 \%$ even for a crystal of class $m \overline{3} m$ (see Table 1). A second problem of DAC measurements is that the diamonds affect the diffraction. In fact, the path inside the diamonds of the incident beam and of the beam diffracted by the crystal is not the same for all reflections, and, therefore, some of them are more attenuated than others. Moreover, the diamonds scatter enormously (coherently and incoherently), but, because they are much larger than the incident beam and they are not centred with respect to the rocking axis, their Bragg reflections are very broad and not so easily predictable on the detector (and therefore not automatically masked by software). Last but not least, the diffraction of diamond produces an additional attenuation of the incident or the diffracted beam (diamond dip) in certain orientations of the DAC (again not easily predictable).

Furthermore, although applying low temperature to DAC is technically possible, the hydrostaticity of the pressure transmission medium could be affected, hampering the very high pressure where the systems may actually show more interesting features. Therefore, ambient temperature is preferable.

A final problem is the powder diffraction rings due to the metal gaskets that incorporate the samples (see Fig. 2). This requires careful modelling of the background.

In order to overcome all these problems, we have planned a careful strategy for data collection, including: (i) two crystals oriented in different ways in the DAC, in order to maximize the data completeness; (ii) a short wavelength, in order to guarantee sufficient coverage at high resolution, despite the inherent shadowing of the DAC; (iii) a highly focused beam, in order to enable separate data collections on two crystals with different orientations, maximizing the signal from the crystal with respect to the scattering of the diamond and avoiding the scattering from the gasket; (iv) sufficient high pressure to minimize the atomic thermal motion. More details about the data collection are given below, and in part were previously reported in Casati et al. (2016).

Of course, other recipes could be adopted, but in general short wavelength and small crystals are mandatory.

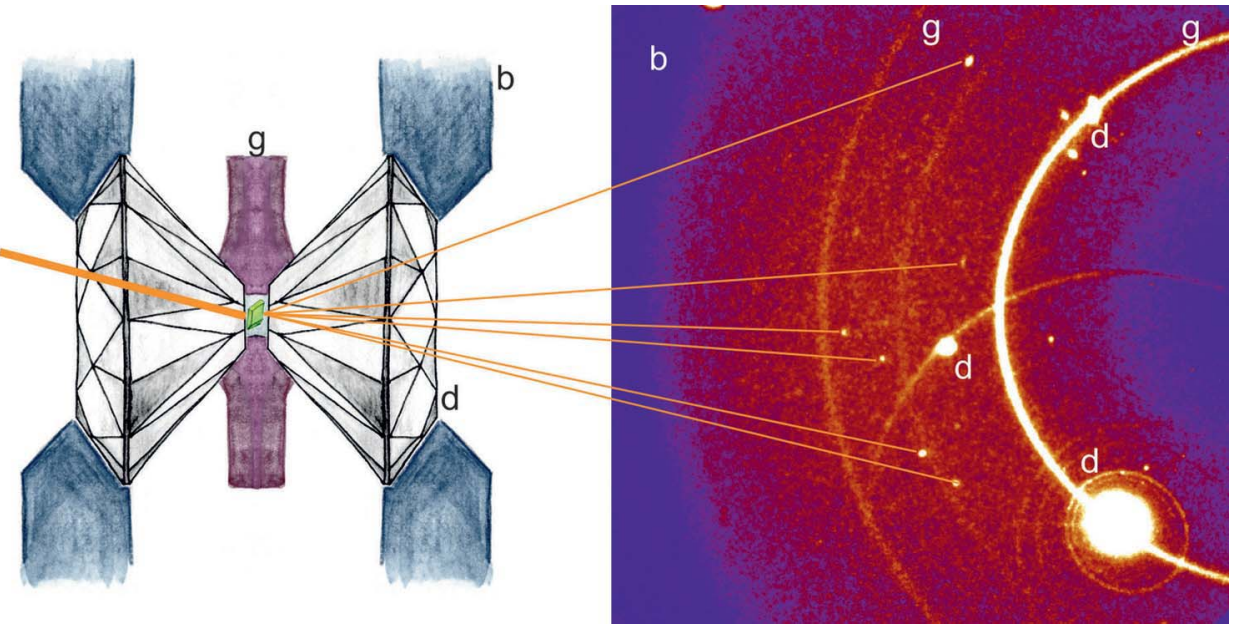

Figure 2

A schematic picture of the diffraction events associated with a diamond anvil cell (modified with permission from Lanza, 2016). The diamonds and their reflections are indicated with 'd', the gasket and the powder rings with 'g', and the DAC body and its shadowing of the diffraction with ' $\mathrm{b}$ '.

\section{Experimental and computational details}

BCA crystallizes in the monoclinic $P 2_{1} / n$ space group, with four molecules in the unit cell. According to the suitability factor $S$ proposed by Stevens \& Coppens (1976), BCA is perfectly ideal for a charge density study. In fact, the suitability $S$ is 3.94 and $2.98 \AA^{3} \mathrm{e}^{-2}$ under ambient conditions and at $7.7 \mathrm{GPa}$, respectively (a higher $S$ value corresponds to a more suitable species for a charge density study). The decreased suitability at higher pressure is due to the smaller unitcell volume, which implies that the information on the same number of 
electrons is spread over a smaller number of reflections, within the same resolution level. This is of course common behaviour for all the species under compression and in general a volume decrease of $20-25 \%$ is expected for organic molecules compressed at $c a$ 7-10 GPa. Nevertheless, the suitability of $\mathrm{BCA}$ at $7.7 \mathrm{GPa}$ remains higher than that of many organometallic molecules and of inorganic phases at ambient pressure (for which $S$ can be even lower than $0.1 \AA^{3} \mathrm{e}^{-2}$ ).

The X-ray crystallographic coordinates and multipolar models for structures reported in this study are given in the supporting information and have been deposited at the Cambridge Crystallographic Data Centre (CCDC), under deposition numbers: 1438912-1438922. In the following, we describe details of the data collection and modelling.

\subsection{Low-temperature data collections}

Single-crystal X-ray diffraction data were collected at several temperatures, up to a resolution of $d=0.67 \AA$, using an Agilent SuperNova diffractometer, equipped with an Mo $K \alpha$ microsource X-ray tube, with an Al filter (Macchi et al., 2011) and mirror optics. An Oxford Cryosystems Cryostream 700 was used for cooling the crystals. The most relevant crystallographic parameters of these data collections are summarized in Table S1 of the supporting information. The data collections at low temperature were useful to monitor the behaviour of the anisotropic displacement parameters of the compound and to compare them with the pressure evolution. Moreover, these experiments did not reveal any anomalous behaviour of the atomic displacement parameters, which indicates that no disorder between two localized configurations occurs. In fact, a hypothetical disorder between two localized configurations of BCA would imply quite large distances between disordered atomic positions, visible in anomalously large atomic displacement parameters, if only an 'average' configuration was refined. In addition, the molecular structure calculated in the crystal at the B3LYP/6-31G(d,p) level (which is inherently ordered) is in almost perfect agreement with the experimental model (see Casati et al., 2016). From these data one can conclude that, at ambient pressure, the molecule of BCA is genuinely based on a delocalized $\pi$ system, also taking into account the accurate very low-temperature electron-density study by Destro \& Merati (1995).

\subsection{High-pressure data collection for charge density}

In keeping with the strategy outlined in $\$ 2$, high-pressure X-ray diffraction experiments were carried out at the I15 beamline of Diamond Light Source using a pinhole defined monochromatic beam with $0.31 \AA$ wavelength, an Oxford Diffraction Atlas CCD detector and a screw-driven-type DAC, equipped with $0.5 \mathrm{~mm}$ culet diamonds DAC. The gasket was indented to $c a 70 \mu \mathrm{m}$, and the crystal samples were loaded in a gasket hole of $250 \mu \mathrm{m}$. The pressure transmission medium was a methanol-ethanol 4:1 $(v / v)$ mixture, which guarantees hydrostaticity up to $c a 10 \mathrm{GPa}$. Several data collections were carried out at different pressures, in order to characterize the pressure dependence of the molecular geometry and inter- molecular packing of BCA. The main results of the model refinements are summarized in Fig. S1 and Table S2 of the supporting information. The charge density data collection was performed at 7.7 GPa (experiment EE7741-1), involving several $\omega$ and $\varphi$ scans at four different $\chi$ positions $(0,30,60$ and $90^{\circ}$ ), using different $\theta$ positions for the detector, in order to measure with the best accuracy the diffraction intensities and to reach the highest resolution $(d=0.5 \AA)$. In the context of this work, accuracy means: (i) high data completeness (i.e. the portion of reciprocal space that is measurable), obtained using two crystals in the DAC; (ii) redundant measures of the diffracted intensities (with every $\omega$ and $\varphi$ scan re-performed with a $2^{\circ}$ offset of $\varphi$ and $\omega$, respectively) in order to minimize random errors and therefore enable a multipolar expansion of the electron density. The chosen high-energy radiation reduces absorption effects and allows the collection of higherorder reflections. A beam smaller than the crystal was selected by a $30 \mu \mathrm{m}$ pinhole, in order to probe one crystal at a time and to maximize the sample/diamond diffraction intensity ratio. Data from the two different crystals were linearly scaled and merged without any weighting scheme. The pressure was calibrated by measuring the fluorescence of two ruby crystals, before and after the measurement.

Data reduction was carried out using the program CrysAlis PRO (Agilent, 2014), excluding the shaded areas from the integration by assigning a well-described vector and opening angle to the cell. Diamond reflections were masked, as described by Casati et al. (2007). Reflections that were outliers in data merging were carefully investigated and rejected if their intensity was significantly lower than their equivalents and lying on the border of the mask or otherwise if their intensity was significantly higher than their equivalents and lying on the tails of a diamond reflection. We rejected reflections on frames collected where a diamond dip occurred, as shown by a transmission scan of the cell in the angular range used. No data correction for absorption of the crystal or of the diamond was applied due to the very short wavelength adopted.

As shown in Table 1 , the best completeness at $d=0.5 \AA$ resolution using a single crystal of $2 / m$ Laue class is ca $46 \%$. Using two crystals with different orientations, and the abovedescribed set-up, we were able to obtain a data completeness of $70 \%$ at $d=0.5 \AA, 89 \%$ at $d=0.83 \AA$ and $91 \%$ at $d=1.2 \AA$. For the sake of comparison, we are reminded that the experiment by Destro \& Merati (1995) at $T=19 \mathrm{~K}$ and ambient pressure was extended up to a resolution of $d=$ $0.44 \AA$ and a data completeness of $54 \%$. This low value is due to the single point detector technique adopted: the data completeness was $100 \%$ for $d>0.77 \AA$, whereas, at higher angles, pre-scanned intensities below 10 counts per s were not measured (see details in Destro, 1988).

\subsection{Multipolar refinement of the data at $7.7 \mathrm{GPa}$}

The multipole refinement was performed using the XD2006 program package (Volkov et al., 2006), which adopts the 
Hansen \& Coppens (1978) formalism to model the electron density using atom-centred multipoles

$$
\rho_{\text {atom }}(\mathbf{r})=\rho_{\text {core }}(\mathbf{r})+P_{\mathrm{v}} \kappa \rho_{\text {valence }}(\kappa \mathbf{r})+\Delta \rho(\mathbf{r})
$$

where

$$
\Delta \rho(\mathbf{r})=\sum_{l=0}^{l_{\max }} \kappa_{l}^{\prime} R_{l}\left(\kappa_{l}^{\prime} r\right) \sum_{m=-l}^{+l} P_{l m} Y_{l m}(\theta, \varphi)
$$

The radial parts of $\rho_{\text {core }}(\mathbf{r})$ and $\rho_{\text {valence }}(\mathbf{r})$ are expressed in terms of Slater-type orbitals computed by means of RoothaanHartree-Fock calculations (Clementi \& Roetti, 1974). Single $\zeta$ orbitals with energy-optimized Slater exponents from Clementi \& Raimondi (1963) are used for the radial part of the deformation terms $\Delta \rho(\mathbf{r})$. The parameters $\kappa$ and $\kappa_{l}^{\prime}$ enable the contraction or expansion of the density shells, whereas $P_{\mathrm{v}}$ and $P_{l m}$ represent the coefficients of the spherical valence and deformation density multipoles, respectively. The angular functions $Y_{l m}$ are density normalized real spherical harmonics.

The function minimized in the least-squares procedure was $\sum_{\mathbf{h}} w_{\mathbf{h}}\left(F_{\mathbf{h}, \text { obs }}-\eta F_{\mathbf{h}, \text { calc }}\right)^{2}$, with the weight $w_{\mathbf{h}}=1 / \sigma^{2}\left(F_{\mathbf{h}, \text { obs }}\right)$. Reflections with $I_{\mathbf{h} \text {,obs }}>2 \sigma_{\mathbf{h} \text {,obs }}\left(I_{\mathbf{h}, \text { obs }}\right)$ were included in the refinement. Given the small crystal samples and the very short wavelength, no extinction correction was necessary. The multipole expansion was truncated at the octupolar level $\left(l_{\max }=3\right)$ for $\mathrm{C}$ and $\mathrm{O}$ atoms. For $\mathrm{H}$ atoms, bond-directed dipoles and quadrupoles were applied. ${ }^{\mathbf{1}}$ The refinement of quadrupoles was possible because the positions of the $\mathrm{H}$ atoms were fixed using values of $\mathrm{C}-\mathrm{H}$ distances computed through a periodic DFT calculation [at the B3LYP/6-31G(d,p) level] at 7.5 GPa and the anisotropic displacement parameters (ADPs) of the $\mathrm{H}$ atoms were determined from the ADPs of the $\mathrm{C}$ and $\mathrm{O}$ atoms, assuming a rigid-body motion of the molecule, using the software SHADE (Madsen, 2006). The coordinates and ADPs of the $\mathrm{H}$ atoms were not refined, thus reducing the number of variables in the refinement.

Several refinement strategies were tested using the abovedescribed model parameters. Owing to the lower data completeness, it was found more appropriate the refine the atomic positions and ADPs of the $\mathrm{C}$ and $\mathrm{O}$ atoms with highangle data ( $\sin \theta / \lambda>0.7 \AA^{-1} ; 163$ parameters). This model was used to calculate the ADPs and positions of $\mathrm{H}$ atoms. All atomic positions and displacement parameters were kept fixed for the final refinement of the multipolar coefficients (300 variables, plus the scale factor). ${ }^{2} \mathrm{H}$ atoms correlated by pseudosymmetry (see Fig. 1) were constrained to have the same set of multipole coefficients. $\kappa$ and $\kappa_{l}^{\prime}$ were refined independently for the $\mathrm{C}$ and $\mathrm{O}$ atoms, using the same $\kappa_{l}^{\prime}$ for dipoles, quadrupoles and octupoles. For the $\mathrm{H}$ atoms, $\kappa$ and $\kappa_{l}^{\prime}$ were fixed at the standard value of 1.2. This refinement produced correlation coefficients below 0.7 for all pairs of

\footnotetext{
${ }^{1}$ In Casati et al. (2016), it is erroneously reported that only dipole functions for $\mathrm{H}$ atoms were used. In the supporting information of that paper, the multipole model is deposited and quadrupole coefficients are clearly reported for $\mathrm{H}$ atoms.

${ }^{2}$ In Casati et al. (2016), it is erroneously reported that there were 302 parameters.
}

variables apart from $\kappa$ and $P_{v}$ of $\mathrm{O} 1$ and $\mathrm{O} 2$ (0.82 in both cases).

The high-order refinement gave a quite satisfactory Hirshfeld (1976) rigid-bond test, with only four bonds exceeding the limit of $1.0 \times 10^{-3} \AA^{2}$ amplitude difference, with a maximum of $1.4 \times 10^{-3} \AA^{2}$ for the two $\mathrm{C}=\mathrm{O}$ bonds. In Destro \& Merati (1995), the Hirshfeld test was of course more successful, with a largest amplitude of $5.4 \times 10^{-4} \AA^{2}$. Since the atomic positions and atomic displacement parameters were fixed during the following refinement steps, the Hirshfeld test remained identical. On the contrary, when the ADPs and the atomic coordinates were refined simultaneously with multipole parameters (464 variables overall, reflection/parameters = 7.7), the Hirshfeld rigid-bond test was much less satisfactory (six bonds exceeded the limit, with differences up to $3.0 \times$ $10^{-3} \AA^{2}$ ), caused by a large correlation between $U^{i j}$ and $\kappa$ parameters, without much improvement of the agreement indices. For these reasons, the model constructed with a highorder refinement of positions and atomic displacement parameters and the subsequent refinement of multipoles was judged to be the most adequate. Noteworthy, this procedure is always recommended to better deconvolute electron density from thermal motion (see also Dos Santos et al. 2014).

\subsection{Wavefunction calculations}

To further investigate the changes in the electron-density distribution of BCA with increasing pressure, wavefunction calculations have also been performed and different computational methods have been exploited to accomplish this task.

First of all, we have carried out traditional X-rayconstrained restricted Hartree-Fock calculations. Since the works by Jayatilaka (1998) and Jayatilaka \& Grimwood (2001), the Hartree-Fock X-ray-constrained wavefunctions (XCW) strategy has emerged as a valid alternative to traditional multipolar expansion models for the reconstruction of electron densities in molecular crystals. As we will see in the following dedicated subsections, the XCW technique practically consists in determining 'experimental ${ }^{3}$ wavefunctions by minimizing the electronic energy of the investigated system under the additional constraint of satisfactorily reproducing a set of experimentally observed X-ray diffraction intensities. Therefore, due to the complementary information provided by the quantum mechanical Hamiltonian of the system, the Jayatilaka method is especially valuable for determining the electron-density distributions of molecular crystals when the $\mathrm{X}$-ray data suffer from incompleteness, as for measurements in diamond anvil cells.

At a second stage, due to the particular interplay between electronic configurations in BCA, we decided to exploit two other wavefunction-based techniques: (i) the strategy devised by Stoll et al. (1980) to determine single Slater determinant wavefunctions constructed with extremely localized molecular orbitals (ELMOs); (ii) the recently developed X-rayconstrained ELMO-valence bond (XC-ELMO-VB) method

\footnotetext{
${ }^{3}$ A large debate is ongoing concerning the meaning of 'experimental' wavefunction; see, for example, Jayatilaka (2012).
} 
(Genoni, 2016, 2017), which can be regarded as a first prototype of the multi-determinant $\mathrm{X}$-ray-constrained wavefunction approach.

The theoretical background of the above-mentioned techniques will be briefly explained in the next subsections. The details and results of the calculations are reported in $\$ 4.2$.

3.4.1. X-ray-constrained restricted Hartree-Fock technique. In the XCW approach (Jayatilaka, 1998; Jayatilaka \& Grimwood, 2001; Grimwood \& Jayatilaka, 2001; Bytheway, Grimwood \& Jayatilaka, 2002; Bytheway et al., 2002; Grimwood et al., 2003; Jayatilaka, 2012), we work with a fictitious non-interacting molecular crystal, in which the wavefunctions describing the different crystal units are formally identical and symmetry related through the unit-cell symmetry operations (i.e. through the roto-translations $\left\{\mathbf{R}_{k}, \mathbf{r}_{k}\right\}_{k=1}^{N_{m}}$, with $N_{m}$ as the number of symmetry-equivalent positions in the unit cell). This allows us to write the global electron-density distribution of the crystal unit cell only in terms of the electron-density $\rho_{0}(\mathbf{r})$ of a reference molecular unit, namely

$$
\rho_{\text {cell }}(\mathbf{r})=\sum_{k=1}^{N_{m}} \rho_{k}(\mathbf{r})=\sum_{k=1}^{N_{m}} \rho_{0}\left(\mathbf{R}_{k}^{-1}\left(\mathbf{r}-\mathbf{r}_{\mathbf{k}}\right)\right)
$$

and, consequently, only in terms of the associated wavefunction $\left|\Psi_{0}\right\rangle$ for the same reference unit. According to the Jayatilaka philosophy, to guarantee that the global electron density of our fictitious non-interacting crystal is identical to the global electron density of the corresponding real interacting system, we look for the wavefunction $\left|\Psi_{0}\right\rangle$ of the reference crystal unit that not only minimizes the corresponding molecular energy, but that also allows the reproduction of as much as possible of a set of experimental structure factor amplitudes. In other words, we seek the wavefunction $\left|\Psi_{0}\right\rangle$ that minimizes the usual Jayatilaka functional

$$
J\left[\Psi_{0}\right]=\left\langle\Psi_{0}|\widehat{H}| \Psi_{0}\right\rangle+\lambda\left(\chi^{2}\left[\Psi_{0}\right]-\Delta\right),
$$

where $\widehat{H}$ is the non-relativistic Hamiltonian operator for the reference unit, $\lambda$ is an external multiplier that gives the strength of the experimental constraints and that is manually adjusted during the calculations, $\Delta$ is the desired agreement between calculated and experimental structure-factor amplitudes, and $\chi^{2}$ is the measure of the statistical agreement between computed and experimental values. Namely, we have

$$
\chi^{2}=\frac{1}{N_{r}-N_{p}} \sum_{\mathbf{h}} \frac{\left(\eta\left|F_{\mathbf{h}}^{\text {calc }}\right|-\left|F_{\mathbf{h}}^{\exp }\right|\right)^{2}}{\sigma_{\mathbf{h}}^{2}},
$$

with $N_{r}$ as the number of considered X-ray diffraction data, $N_{p}$ as the number of adjustable parameters, $\mathbf{h}$ as the triads of Miller indices labelling the reflections, $\sigma_{\mathbf{h}}$ as the experimental error associated with the observed structure-factor amplitude $\left|F_{\mathbf{h}}^{\exp }\right|$ and $\eta$ as a scale factor that is determined to minimize $\chi^{2}$.

In the original and standard XCW-restricted Hartree-Fock approach (Jayatilaka, 1998; Jayatilaka \& Grimwood, 2001; Grimwood et al., 2003), the chosen ansatz for the wavefunction $\left|\Psi_{0}\right\rangle$ for the reference crystal unit is a simple single Slater determinant of doubly occupied molecular orbitals. Starting from this ansatz, it is possible to show that finding the wave- function $\left|\Psi_{0}\right\rangle$ that minimizes functional (4) is equivalent to solving the following modified Hartree-Fock equations

$$
\widehat{F}^{J}\left|\varphi_{k}\right\rangle=\epsilon_{k}\left|\varphi_{k}\right\rangle,
$$

where the Fock-Jayatilaka operator $\widehat{F}^{J}$ is

$$
\widehat{F}^{J}=\widehat{F}^{I}+\lambda_{J} \sum_{\mathbf{h}} K_{\mathbf{h}} \operatorname{Re}\left\{F_{\mathbf{h}}^{\text {calc }}\right\} \hat{I}_{\mathbf{h}, R}+\lambda_{J} \sum_{\mathbf{h}} K_{\mathbf{h}} \operatorname{Im}\left\{F_{\mathbf{h}}^{\text {calc }}\right\} \hat{I}_{\mathbf{h}, c}
$$

with $\widehat{F}$ as the usual Fock operator and with the multiplicative constant $K_{\mathbf{h}}$ expressed like this

$$
K_{\mathbf{h}}=\frac{2 \eta}{N_{r}-N_{p}} \frac{\eta\left|F_{\mathbf{h}}^{\mathrm{calc}}\right|-\left|F_{\mathbf{h}}^{\mathrm{obs}}\right|}{\sigma_{\mathbf{h}}^{2}\left|F_{\mathbf{h}}^{\mathrm{calc}}\right|} .
$$

Finally, $\hat{I}_{\mathbf{h}, R}$ and $\hat{I}_{\mathbf{h}, c}$ are both hermitian operators corresponding to the real and imaginary parts of the structurefactor operator, respectively, which can be expressed as

$$
\hat{I}_{\mathbf{h}}=\hat{I}_{\mathbf{h}, R}+i \hat{I}_{\mathbf{h}, C}=\sum_{k=1}^{N_{m}} e^{i 2 \pi\left(\mathbf{R}_{k} \mathbf{r}+\mathbf{r}_{\mathbf{k}}\right) \cdot(\mathbf{B h})}
$$

with $\mathbf{B}$ as the matrix of the reciprocal-lattice.

3.4.2. The unconstrained extremely localized molecular orbitals method. The traditional ELMO technique proposed by Stoll et al. (1980) can be considered as a natural evolution of the pioneering group function method introduced by McWeeny since the 1960s (McWeeny, 1959, 1960, 1992). Furthermore, it is also strictly related to all those theoretical strategies that are mainly aimed at expressing the global electronic wavefunction of a system in terms of functions describing its smaller components (Adams, 1961; Huzinaga \& Cantu, 1971; Gilbert, 1974; Matsuoka, 1977; Smits \& Altona, 1985; Francisco et al., 1992; Ordejón et al., 1993; Couty et al., 1997; Fornili et al., 2003; Szekeres \& Surján, 2003).

Following Stoll, we assumed a closed-shell molecule composed of $2 \mathrm{~N}$ electrons and we introduced a localization scheme that subdivides the system into $f$ subunits (e.g. atoms, bonds and functional groups) that may overlap. We afterwards assign a local basis-set $\beta^{i}=\left\{\left|\chi_{\mu}^{i}\right\rangle\right\}_{\mu=1}^{M^{i}}$ for each fragment, which is the set of basis functions centred on the atoms belonging to each subunit. Consequently, we expand the ELMOs corresponding to the generic $i$ th fragment over $\beta^{i}$ and we write the generic $\alpha$ th ELMO as

$$
\left|\varphi_{\alpha}^{i}\right\rangle=\sum_{\mu=1}^{M^{i}} C_{\mu \alpha}^{i}\left|\chi_{\mu}^{i}\right\rangle .
$$

According to Stoll, the wavefunction describing the $2 \mathrm{~N}$-electron closed-shell system under examination is the single Slater determinant constructed with the extremely localized molecular orbitals localized on the different subunits in which we have previously subdivided the molecule of interest

$$
\left.\left|\Psi_{E L M O}\right\rangle=\mathcal{M} \hat{A}\left[\varphi_{1}^{1} \bar{\varphi}_{1}^{1} \ldots \varphi_{n_{1}}^{1} \bar{\varphi}_{n_{1}}^{1} \ldots \varphi_{1}^{f} \bar{\varphi}_{1}^{f} \ldots\right\} \varphi_{n_{f}}^{f} \bar{\varphi}_{n_{f}}^{f}\right],
$$

where $\hat{A}$ is the antisymmetrizer, $n_{i}$ is the number of occupied ELMOs for the $i$ th fragment and $\bar{\varphi}_{\alpha}^{i}$ is a spin orbital with 
spatial part $\varphi_{\alpha}^{i}$ and spin part $\beta$, and $\mathcal{M}$ is a normalization constant which can be expressed as follows

$$
\mathcal{M}=\frac{1}{\sqrt{(2 N) ! \operatorname{det}[\mathbf{S}]}}
$$

with $\operatorname{det}[\mathbf{S}]$ as the determinant of the overlap matrix between the occupied ELMOs.

As the traditional Hartree-Fock molecular orbitals are obtained by minimizing the energy of the single Slater determinant built up with them, in the same way, the ELMOs are determined by variationally minimizing the energy corresponding to wavefunction (11). This is actually equivalent to solving the following eigenvalue equation for each fragment

$$
\widehat{F}^{i}\left|\varphi_{\alpha}^{i}\right\rangle=\varepsilon_{\alpha}^{i}\left|\varphi_{\alpha}^{i}\right\rangle
$$

where $\widehat{F}^{i}$ is the modified Fock operator for the generic $i$ th subunit, namely

$$
\widehat{F}^{i}=\left(1-\widehat{\rho}+\widehat{\rho}^{i \dagger}\right) \widehat{F}\left(1-\widehat{\rho}+\widehat{\rho}^{i}\right),
$$

with $\widehat{F}$ as the usual Fock operator, $\hat{\rho}$ as the local density operator for fragment $i$, which depends only on the occupied ELMOs of the subunit, and $\widehat{\rho}$ as the global density operator, which couples the eigenvalue equations associated with the different fragments because it depends on all the occupied ELMOs of the molecule under exam.

Therefore, by solving equation (13) it is really possible to determine molecular orbitals that are strictly localized on chemically meaningful fragments (atoms, bonds or functional groups) and, consequently, to obtain single Slater determinant wavefunctions that are closer to the usual traditional chemical concepts, as that of chemical resonance structure (see \$3.4.3).

For the sake of completeness, it is also worth noting that the original ELMO technique has also been recently extended in the framework of the X-ray-constrained wavefunction strategy, giving rise to the new XC-ELMO method that allows the extraction of extremely localized molecular orbitals from experimental structure-factor amplitudes (Genoni, 2013a,b; Dos Santos et al., 2014; Genoni \& Meyer, 2016). However, in this work, X-ray-constrained ELMO calculations have not been used to investigate the partial rupture of aromaticity in BCA at high pressures.

3.4.3. The X-ray-constrained ELMO valence bond method technique. As mentioned in \$3.4.1, the traditional XCW strategy works within the approximation of the single Slater determinant wavefunction ansatz, which is probably the main reason why the Jayatilaka method captures only partially the electron correlation effects on the electron density (Genoni et al., 2017). The novelty of the new X-ray-constrained ELMOvalence bond technique (Genoni, 2016, 2017) consists in the fact that a multi-determinant wavefunction ansatz has been considered for the first time in the framework of the Jayatilaka approach. In particular, in the XC-ELMO-VB method, the wavefunction $\left|\Psi_{0}\right\rangle$ for the reference molecular unit is written as follows

$$
\left|\Psi_{0}\right\rangle=\left|\Psi_{\text {XC-ELMO-VB }}\right\rangle=\sum_{j} C_{j}\left|\Psi_{j}\right\rangle
$$

where the wavefunctions $\left|\Psi_{j}\right\rangle$ are Slater determinants associated with the possible resonance structures of the system under investigation. In particular, in the novel XC-ELMOVB strategy, they are normalized ELMO wavefunctions resulting from simple unconstrained ELMO calculations exploiting localization schemes that correspond to the different resonance structures of the system under study. For instance, for the case studied in this paper (the syn-1,6:8,13biscarbonyl[14]annulene molecule), the wavefunction $\left|\Psi_{\mathrm{XC}-\mathrm{ELMO}-\mathrm{VB}}\right\rangle$ has been written as a linear combination of two ELMO wavefunctions: (i) one obtained by imposing a localization scheme with the fragments for the $\pi$ electrons corresponding to bonds $\mathrm{C} 14-\mathrm{C} 1, \mathrm{C} 2-\mathrm{C} 3, \mathrm{C} 4-\mathrm{C} 5, \mathrm{C} 6-\mathrm{C} 7$, $\mathrm{C} 8-\mathrm{C} 9, \mathrm{C} 10-\mathrm{C} 11$ and $\mathrm{C} 12-\mathrm{C} 13$ (resonance structure $A$ of BCA, see Fig. 1a); (ii) and the other determined by exploiting a localization scheme with the fragments for the $\pi$ electrons corresponding to bonds $\mathrm{C} 1-\mathrm{C} 2, \mathrm{C} 3-\mathrm{C} 4, \mathrm{C} 5-\mathrm{C} 6, \mathrm{C} 7-\mathrm{C} 8$, $\mathrm{C} 9-\mathrm{C} 10, \mathrm{C} 11-\mathrm{C} 12$ and $\mathrm{C} 13-\mathrm{C} 14$ (resonance structure $B$ of BCA, see Fig. 1b).

In the current version of the XC-ELMO-VB method, the predetermined unconstrained ELMO wavefunctions (or, in other words, the pre-computed unconstrained extremely localized molecular orbitals) are kept frozen and, therefore, the new XCW techniques practically consists of determining the coefficients $\left\{C_{j}\right\}$ of linear combination (15) that minimize functional (4).

However, since the ELMO Slater determinants in expansion (15) are non-orthogonal between each other, the coefficients $\left\{C_{j}\right\}$ do not give the weights of the associated resonance structures directly. The real weights are actually obtained by computing the Chirgwin-Coulson coefficients (Chirgwin \& Coulson, 1950) $\left\{K_{j}\right\}$

$$
K_{j}=\left|C_{j}\right|^{2}+\sum_{m \neq j} C_{j} C_{m} S_{j m},
$$

with $S_{j m}=\left\langle\Psi_{j} \mid \Psi_{m}\right\rangle$ as the overlap integral between the precomputed unconstrained ELMO wavefunctions $\left|\Psi_{j}\right\rangle$ and $\left|\Psi_{m}\right\rangle$.

In all the XC-ELMO-VB methods, the adjustable parameter $\lambda$ is iteratively varied until the weights associated with the resonance structures converge, in particular, until when the largest absolute variation of the Chirgwin-Coulson coefficients between two consecutive $\lambda$ steps is lower than $1.0 \times$ $10^{-3}$.

\section{Results and discussion}

As reported in Casati et al. (2016), the most evident feature of the high-pressure structure forms of BCA is the asymmetrization of the molecular geometry that significantly deviate from the ideal $C_{2 v}$ symmetry observed at ambient pressure, and produces an inhomogeneous contraction of the $\mathrm{C}-\mathrm{C}$ bonds of the annulene skeleton (see Table S2 of the supporting information). From the geometrical point of view, this can be rationalized as a loss of the perfect resonance 
between configurations $A$ and $B$ (Fig. 1) and a partial localization of one of them (namely $A$ ). The purpose of a charge density investigation was to analyse this very peculiar behaviour from the point of view of the electron-density accumulation in the bonds. As reported in Casati et al. (2016), many indicators, including the electron density at the bond critical points (Bader, 1990) and the electron delocalization indices (Bader \& Beddall, 1972) calculated from an X-ray-constrained wavefunction, indicate that there is a partial localization of one of the two resonance forms.

The interpretation of the pressure-induced modifications is not discussed here further. In the following paragraphs, instead, we analyse in detail the electron-density models refined or calculated for $\mathrm{BCA}$ at $7.7 \mathrm{GPa}$, in order to assess the quality of the measurement and the significance of the extracted information. First, we report an analysis of the multipolar model, which also provides the basis for the X-rayconstrained wavefunction calculations. Then we will analyse the results of the various wavefunction methods, particularly focusing on the results obtained with the new XC-ELMO-VB strategy.

\subsection{Analysis of the refined multipolar model}

4.1.1. The model flexibility. This topic is of fundamental importance in multipolar expansion, because the atomcentred modelling of equation (1) implies a number of restrictions that may affect the results. The flexibility depends on three main factors: $(a)$ the atomic shells which are refined; (b) the radial functions; $(c)$ the angular functions.

The standard multipolar model implies a rigid core electron density and a refined valence shell. The population of the core is fixed and its radial function generally coincides with the core orbitals ( $1 s$ orbitals for the $\mathrm{C}$ and the $\mathrm{O}$ atoms in $\mathrm{BCA}$, for example) of the Roothaan-Hartree-Fock atomic wavefunctions, such as those proposed by Clementi \& Roetti (1974). Recently, an extended Hansen and Coppens model was proposed (Fischer et al., 2011), in which the core population and its radial function can also be refined. This procedure is similar to a strategy implemented in the program VALRAY (Stewart et al., 2000), although all atoms of a given element type usually share the same core parameters, thus resulting in a rather rigid model. This was in fact the model adopted by Destro \& Merati (1995). The quality of the high-pressure data does not allow any 'shell' flexibility, which is also very difficult for data collected under ambient conditions. Therefore, the refinement of BCA data is limited to the $K$ shell of the $\mathrm{H}$ atoms and to the $L$ shell of the $\mathrm{C}$ and $\mathrm{O}$ atoms, which is rather a typical multipolar refinement. In order to increase the data/ parameter ratio, the $\mathrm{H}$ atoms were refined with a pseudosymmetry constraint: only three of them were freely refined (namely $\mathrm{H} 2, \mathrm{H} 3$ and $\mathrm{H} 7$; see Fig. 1), while the pseudoequivalent ones were constrained to share the same set of multipoles.

Apart from the treatment of the core, the radial functions of the valence orbitals can also be flexible, refining the $\kappa$ parameters of the multipolar expansion (1). The spherical valence is normally constructed by all valence orbital functions of a Roothaan-Hartree-Fock expansion. When a single $\kappa$ is adopted for the valence monopole, it implies that both the $2 s$ and $2 p$ orbitals contract or expand in the same way. On the contrary, for higher multipoles, the radial functions are normally single $\zeta$ Slater orbitals (Clementi \& Raimondi, 1963). In principle, an individual scaling is possible for each pole $l$ (or even for each $m_{l}$ function), but this would produce a divergent refinement and only one $\kappa^{\prime}$ is typically refined in standard models, sometimes even applying a $\kappa=\kappa^{\prime}$ constraint (Abramov et al., 1999) to avoid divergence or unphysical results, like a very diffuse valence shell of terminal atoms in a molecule. In our best model, we refined $\kappa$ and $\kappa^{\prime}$ separately for the $\mathrm{C}$ and $\mathrm{O}$ atoms, but only one set of radial scaling was adopted for each element type. In fact, the two $\mathrm{O}$ atoms are of the same kind and also correlated by the molecular pseudosymmetry $\left(C_{2}, C_{s}\right.$ or $C_{2 v}$ ). On the contrary, all the $\mathrm{C}$ atoms are of $s p^{2}$-type and involved in the aromatic ring or in the carbonyl bonds, therefore normally only one $\kappa$ set should be adopted. In fact, a tentative flexibility with the four 'unique' $\mathrm{C}$ atoms in $C_{2 v}$ pseudosymmetry (or seven unique atoms in $C_{2}$ or $C_{s}$ pseudosymmetry) would result in a refinement which is too flexible and unstable. No radial scaling was adopted for the $\mathrm{H}$ atoms.

Concerning the angular flexibility, the order of the multipole expansion is normally fixed by the number of reflections usable for refining such quantities. Noteworthy, only lowresolution data actually contain information on the valence electron-density distribution. A drawback of high pressure is that the sampling of the low resolution is less dense, due to the volume contraction, and therefore the number of usable reflections to refine these parameters is lower. In fact, at the resolution $d=0.71 \AA\left(\sin \theta / \lambda=0.7 \AA^{-1}\right.$, where the scattering of the $L$ shell of $\mathrm{O}$ and $\mathrm{C}$ atoms becomes negligible), the number of theoretical reflections at $0.0 \mathrm{GPa}$ is 3374 , whereas at $7.7 \mathrm{GPa}$ it is 2638 (of which, only 2236 were measured). This is the reason why the model was refined in two blocks: $(a)$ coordinates and ADPs using the high-resolution data only (2455 reflections for 163 parameters) and (b) multipolar coefficients and radial scaling (using all data, but with 1863 'effective' low-angle reflections for 301 parameters).

In a standard multipolar model, $\mathrm{H}$ atoms are expanded only up to a dipolar level. Quadrupoles are refinable only if the anisotropic displacement parameters are also available (for example, from neutron diffraction or from rigid-body motion calculations). Thanks to the estimation of the H-atom ADPs made through SHADE (Madsen, 2006), the multipolar model of BCA could be extended up to quadrupolar functions for the $\mathrm{H}$ atoms, although only one dipole $(z)$ and one quadrupole $\left(3 z^{2}-1\right)$ were refined $(z$ being the local coordinate coinciding with the $\mathrm{C}-\mathrm{H}$ bond direction). Second row elements are usually treated at least up to $l=3$. Octupoles are in fact fundamental for the treatment of $s p^{2}$-hybridized $\mathrm{C}$ or $\mathrm{O}$ atoms (see, for example, Macchi, 2013). For the $\mathrm{C}$ and $\mathrm{O}$ atoms, a full set of multipoles could be refined up to the octupolar level without chemical constraints.

For the sake of comparison, we note that Destro \& Merati (1995), using the low-temperature accurate data, could 
simultaneously refine the atomic positions, displacements and multipoles, using up to 385 electron-density parameters (instead of 301 in our model) and 163 position/atomic displacement parameters and scale factor (coinciding with our model). The 'effective' number of reflections for the electrondensity modelling was 2963 . Thus, the effective reflections/ parameters ratio for the multipoles was 7.7 (= 2963/385), compared with $6.2(=1863 / 301)$ in our refinement. Destro \& Merati (1995) also fixed the positions and atomic displacement parameters of the $\mathrm{H}$ atoms in the final model, using the generalized scattering factor approach (Stewart, Bentley \& Goodman, 1975) implemented in $V A L R A Y$ to initially refine the positions, and they adopted an approach very similar to ours for the treatment of atomic displacement parameters.

It is noteworthy, given the constraints applied to $\mathrm{H}$ atoms and to the radial scaling, that the parameters that describe deviations from the $C_{2 v}$ pseudosymmetry of the molecule are the coordinates, ADPs and multipoles of the $\mathrm{C}$ atoms.

4.1.2. Atomic displacement parameters. As introduced above, a general requirement of charge density studies is quenching the thermal motion in order to allow an easier deconvolution of the electron density. This is in keeping with the Born-Oppenheimer and the adiabatic approximation, i.e. the electron density is only a function of the nuclear configuration and it is not deformed along the coordinates of a vibrational mode. Of course, the temperature to achieve this condition strongly depends on the investigated species: for an organic molecular crystal, only low ( $c$ a $100 \mathrm{~K}$ ) or even very low $(<5-20 \mathrm{~K})$ temperatures are necessary, whereas for a hard material, room temperature (or even above) is sufficient. One may take the Debye temperature $\Theta_{D}$ (the highest temperature that can be achieved due to a single normal vibration) as a reference to evaluate a sensible limit $\left(T<\frac{1}{2} \Theta_{\mathrm{D}}\right.$; Larsen, 1995). Anyway, this assumption holds true only at ambient pressure. On the contrary, for crystals compressed at high pressure, the reduced space available forces the atoms to reduce the amplitude of their motion, without reducing their thermal energy. As a result, the atomic anisotropic displacement parameters decrease like at low $T$ and consequently the diffraction increases, especially at a high diffraction angle, in keeping with the Debye-Waller formalisms.

The data collected at different pressures or temperatures enabled us to evaluate the atomic displacement parameters as a function of the thermodynamic variables. In Fig. 3, we report a comparison between averaged atomic $U_{\text {eq }}$ obtained from low $T$ or from high-pressure experiments. It is interesting to observe that $U_{\text {eq }}=f(P)$ or $U_{\text {eq }}=f\left(T^{-3}\right)$ behave very similarly, which leads us to conclude that a high-pressure experiment on BCA at $P=7.7 \mathrm{GPa}$ is similar (in terms of thermal motion) to an experiment carried out at $T=123 \mathrm{~K}$, i.e. at a temperature suitable for standard charge density data collections, although at the upper limit.

Of course, this conclusion cannot be generalized, because the decrease in atomic thermal motion is not only a function of the pressure, but also of the crystal compressibility. BCA lacks any strong intermolecular interaction and is therefore very compressible. For a system having both strong and weak intermolecular interactions, the same assumption could not be guaranteed. In fact, the strong intermolecular interactions would make the solid not much compressible; therefore, the weakly bound part of the crystal will still move significantly even at high pressure.

4.1.3. Residuals and agreement indices. A crystallographic structural model, as well as an electron-density model, is normally judged from the agreement indices $R\left(R_{1}\right.$ and $\left.w R_{2}\right)$ and residual electron densities, whereas the quality of the data is judged from the internal agreement of the equivalent intensities of the crystal Laue class $\left(R_{\text {int }}\right)$. For our experiment in a DAC and the planned strategy, some warnings are necessary. First of all, the data completeness was obtained after merging data sets collected on two differently oriented crystals. This inherently produces lower precision and a higher $R_{\text {int }}$. Moreover, the very fine focused beam and small size of the crystals implies a critical reproducibility of equivalent intensities. For this reason, it is expected that the precision of the measurements is significantly lower than in standard charge density data collections. In addition, for synchrotron data collections, it is rather normal to obtain larger $R_{\text {int }}$ values (see, for example, Morgenroth et al., 2008), due to beam instabilities and other effects that may compromise a perfect reproducibility. This suggests that a large redundancy is a must in order to mediate all the effects. Last, but not least, the very small size of the crystal samples implies very weak diffraction at high angle. It is therefore not surprising that $R_{\text {int }}$ is large for the data collection at $7.7 \mathrm{GPa}$; see Table 2 which summarizes the crystallographic data for this measurement. The same arguments also hold for the agreement indices, which are

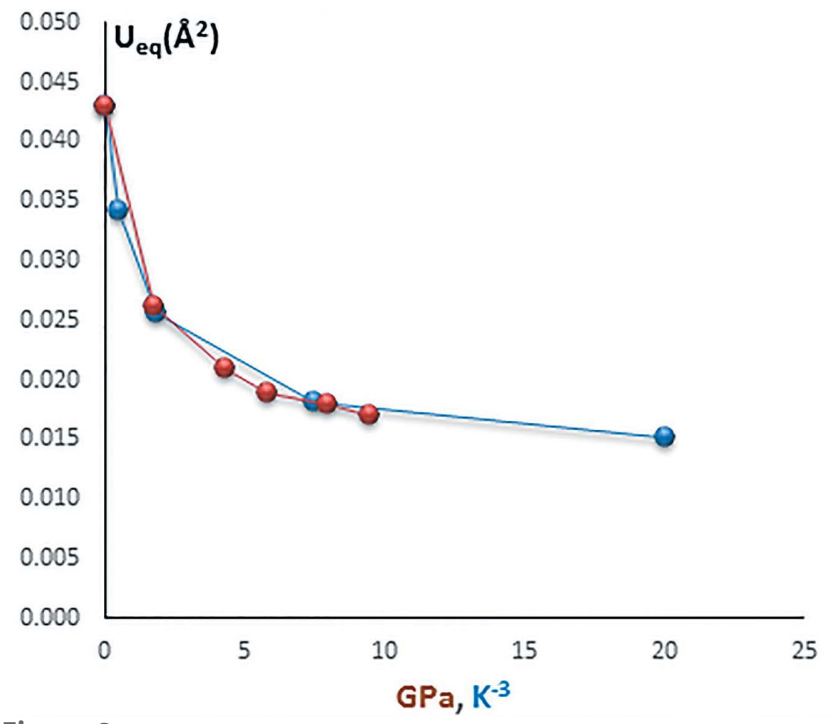

Figure 3

The average values of refined atomic $U_{\text {eq }}$ values $\left(\AA^{2}\right)$ of $\mathrm{C}$ and $\mathrm{O}$ atoms in BCA from experiments at variable pressure (red) or temperature (blue). The abscissa is Pressure (GPa, for the red points) or the function $f(T)=1.5 \times 10^{7}\left(1 / T^{3}-1 / 298^{3}\right)\left(\mathrm{K}^{-3}\right.$, for the blue points $)$, respectively. For $T=123$ and $90 \mathrm{~K}, f(T)=7.5$ and 20 , respectively. If the correlation $f(T)$ versus $P$ holds true also at higher $P$ and lower $T$, a temperature of $20 \mathrm{~K}$ would correspond to a pressure of $c a 2 \mathrm{TPa}(2000 \mathrm{GPa})$. This implies that in the pressure range of measurable single-crystal X-ray diffraction experiments (namely, 0-20 GPa), the atomic displacement parameters of $\mathrm{BCA}$ are always in the range of a liquid-nitrogen temperature. 
Table 2

Selected crystallographic data and refinement parameters for the extensive data set collected at $7.7 \mathrm{GPa}$ and used for the electron-density refinement.

\begin{tabular}{ll}
\hline$a(\AA)$ & $8.6187(3)$ \\
$b(\AA)$ & $11.5861(6)$ \\
$\mathrm{c}(\AA)$ & $8.6105(3)$ \\
$\beta\left({ }^{\circ}\right)$ & $92.788(3)$ \\
$V\left(\AA^{3}\right)$ & $858.80(6)$ \\
Total reflections, unique, observed $[I>2 \sigma(I)]$ & $23698,5047,3554$ \\
$R_{\text {int }}($ all data) & 0.116 \\
$R_{\text {int }}[I>2 \sigma(I)]$ & 0.079 \\
Number of parameters for the & 163 \\
$\quad$ spherical atom refinement $\dagger$ & \\
& \\
$R_{1}[I>2 \sigma(I)]$ & \\
$\sin \theta / \lambda<0.7 \AA^{-1}$ & 0.073 \\
$\sin \theta / \lambda>0.7 \AA^{-1}$ & 0.103 \\
All data & 0.089 \\
$\Delta \rho_{\min }, \Delta \rho_{\max }\left(\mathrm{e} \AA^{-3}\right)$ & $-0.44,0.60$ \\
$\sin \theta / \lambda<0.7 \AA^{-1}$ & $-0.60,0.88$ \\
All data & \\
Total number of parameters for & 464 \\
the multipolar model $\ddagger$ & \\
& \\
$R_{1}[I>2 \sigma(I)]$ & \\
$\sin \theta / \lambda<0.7 \AA^{-1}$ & 0.0451 \\
All data & 0.0676 \\
$\Delta \rho_{\min }, \Delta \rho_{\max }\left(\mathrm{e} \AA^{-3}\right)$ & \\
$\sin \theta / \lambda<0.7 \AA^{-1}$ & $-0.30,0.29$ \\
All data & $-0.53,0.55$ \\
\hline
\end{tabular}

$\dagger$ Using the $X D 2006$ conventions and $w_{h}=1 / \sigma^{2}\left(F_{h, \text { obs }}^{2}\right)$. $\ddagger$ The refinement was carried out in two steps: (1) high-order spherical atom refinement of the positional and atomic displacement parameters for $\mathrm{C}$ and $\mathrm{O}$ atoms (163 parameters); (2) refinement of the multipole coefficients using all reflections (302 parameters). A scale factor was refined in both cases. certainly larger than for standard charge density models, though not unusual for synchrotron data collections (see Morgenroth et al., 2008). On the other hand, analysis of the residual electron densities is quite promising. In particular, two functions are important: the experimental deformation density (i.e. the Fourier transformation of the difference between the measured structure factors and those calculated with spherical atoms) and the residual electron density after the multipolar refinement. The experimental deformation density emphasizes the electron density in the bonds, which is not described by a spherical atom model. In Fig. 4 we see that larger residuals occur in the middle of the $\mathrm{C}-\mathrm{C}$ bonds, indicating that the data contain in fact the expected information. This would be a normal observation for standard electrondensity studies, but it is a surprisingly good result for a highresolution data collection in a DAC. The residuals after multipolar analysis are also very promising, because they are not enormous $\left(+0.55 ;-0.53\right.$ e $\AA^{-3}$, using all data), although certainly higher than what was expected under standard conditions. If analysed with the method proposed by Meindl \& Henn (2008), the distribution of the residuals shows an almost perfect Gaussian distribution, thus indicating no systematic effects, in keeping with the residual map being noisy but without systematic features. Therefore, although the agreement indices of the multipolar model are not excellent and quite large compared with standard charge density data collections, the effect on the refined multipolar model is not so dramatic.

For the sake of comparison, we note that the model by Destro \& Merati (1995) returned $R_{1}=0.012$ for $I>2 \sigma(I)$ and $d$ $>0.78 \AA$ (the $\mathrm{Cu} K \alpha$ sphere, within which all data were collected), compared with 0.042 for our model at the same resolution (with $87 \%$ data completeness). A comparison at higher resolution is not possible, given the strategy adopted by Destro \& Merati (1995) for the data collection (see above), which limited the measurement to the most intense reflections only. No residual electron density was reported in that study; anyway, using the deposited data and a consistent model, $\Delta \rho_{\min , \max }=$ \pm 0.23 e $\AA^{-3}$ was calculated.

\subsection{Analysis of the wavefunction calculations}

Because of the data incompleteness and the rather low precision of the data, it was mandatory to double check the quality of the electron density of the multipolar model (and its interpretation) also using a wavefunction-based

(a) The experimental deformation density map, (b) the residual electron-density map after multipolar refinement, $(c)$ the static deformation map obtained from the multipolar refinement and $(d)$ the distribution of residuals. The isosurface values are $\pm 0.35 \mathrm{e}^{-3}$ (positive values are green, negative values are red). 
method, as explained in the experimental section. For this reason, we first computed a standard X-ray-constrained molecular wavefunction (Jayatilaka \& Grimwood, 2001) for BCA at $7.7 \mathrm{GPa}$ and then tested the new methods with localized schemes.

4.2.1. X-ray-constrained restricted Hartree-Fock computations. The molecular orbital calculations were carried out by means of the TONTO package (Jayatilaka \& Grimwood, 2003) at the Hartree-Fock level with the 6-31G(d,p) basis set, using a constraint to the experiment up to $\lambda_{\max }=1$ [see equation (4)], after verifying that no significant improvement in $\chi^{2}$ could be obtained. Table 3 summarizes the results of the fitting, showing a similar kind of agreement as for the multipolar model. As reported in Casati et al. (2016), the electron density calculated from this wavefunction enabled the partial localization of the double bond character in the configuration $A$ to be confirmed (Fig. 1). An advantage of this electrondensity reconstruction is being less subject to the noise of the diffraction data than the multipolar model. Noteworthy, given the necessity to separate the multipole variables from coordinates and ADPs (refined only at high angle), the X-rayconstrained wavefunction calculation is a perfect alternative to the multipolar refinement. An additional advantage of a wavefunction determination is the possibility of calculating not only the one-electron density but also the density matrices and, therefore, the electron delocalization indices. This model is not further discussed here, because the focus of this work was in fact finding additional information from localized orbital models and pseudo valence bond treatment, as discussed in the next two paragraphs.

4.2.2. Unconstrained ELMO calculations. We have performed unconstrained ELMO calculations using (i) the crystallographic structure of BCA determined at ambient pressure and low temperature (19 K; Destro \& Merati, 1995) and (ii) the crystal structure determined more recently at 7.7. GPa by Casati et al. (2016).

In particular, for each structure we have carried out three different unconstrained ELMO calculations corresponding to three different localization schemes (see Fig. 5) for the $\pi$ electrons of the 14-carbon atom ring of the molecule: (i) a localization scheme with a unique fragment completely delocalized on the $14 \mathrm{C}$ atoms (from now on indicated as DScheme); (ii) a localization scheme with the fragments for the
Table 3

Results of the X-ray-constrained wavefunction calculations.

\begin{tabular}{ll}
\hline$R_{1}[I>2 \sigma(I)]$ & \\
$\quad \sin \theta / \lambda<0.7 \AA^{-1}$ & 0.055 \\
All data & 0.066 \\
& \\
$\chi^{2}[I>2 \sigma(I)]$ & 1.42 \\
$\sin \theta / \lambda<0.7 \AA^{-1}$ & 2.33 \\
$\quad$ All data & \\
\hline
\end{tabular}

$\pi$ electrons corresponding to bonds $\mathrm{C} 14-\mathrm{C} 1, \mathrm{C} 2-\mathrm{C} 3, \mathrm{C} 4-$ $\mathrm{C} 5, \mathrm{C} 6-\mathrm{C} 7, \mathrm{C} 8-\mathrm{C} 9, \mathrm{C} 10-\mathrm{C} 11, \mathrm{C} 12-\mathrm{C} 13$ (from now on indicated as A-Scheme) and corresponding to resonance structure $A$ of BCA in Fig. 1; (iii) a localization scheme with the fragments for the $\pi$ electrons corresponding to bonds $\mathrm{C} 1-$ $\mathrm{C} 2, \mathrm{C} 3-\mathrm{C} 4, \mathrm{C} 5-\mathrm{C} 6, \mathrm{C} 7-\mathrm{C} 8, \mathrm{C} 9-\mathrm{C} 10, \mathrm{C} 11-\mathrm{C} 12, \mathrm{C} 13-$ $\mathrm{C} 14$ (from now on indicated as B-Scheme) and corresponding to resonance structure $B$ of BCA in Fig. 1. For the sake of completeness, we point out that a localization scheme corresponding to the traditional Lewis structure has been adopted for the remaining part of the molecule (i.e. core, lone pairs, $\sigma$ and $\pi_{\mathrm{C}=\mathrm{O}}$ electrons). Furthermore, three different basis-sets have been considered for our calculations: 6-31G, 6-31G(d,p) and cc-pVDZ.

After performing the calculations described above, for each localization scheme, we have compared the unconstrained ELMO energies obtained for the ambient and the high-pressure structures. In other words, we have determined the following energy differences:

$$
\Delta E_{X}=E_{\mathrm{ELMO}-X}^{\mathrm{HP}}-E_{\mathrm{ELMO}-X}^{\mathrm{AP}}
$$

where $X$ indicates the localization scheme (hence, $X=\mathrm{D}, \mathrm{A}$ or B) and where HP and AP are 'high pressure' and 'ambient pressure', respectively.

The obtained values, which are collected in Table 4, show that a completely delocalized electronic structure is more energetically favourable at ambient pressure than at high pressure. Furthermore, in line with this result, we can also observe that for the electronic configurations represented by localization schemes $A$ and $B$, we have opposite trends when external pressure increases. In fact, while configuration $A$ becomes more favourable at high pressure, at $7.7 \mathrm{GPa}$, configuration $B$ is significantly less stable from the energetic point of view. All these results clearly suggest that, at high

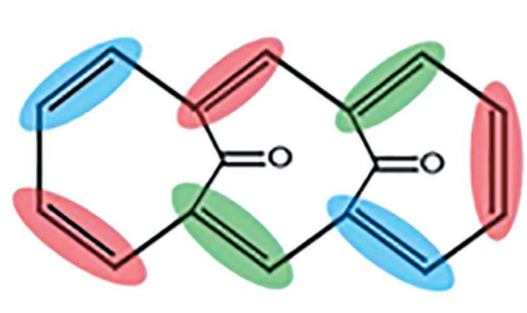

$\boldsymbol{A}$

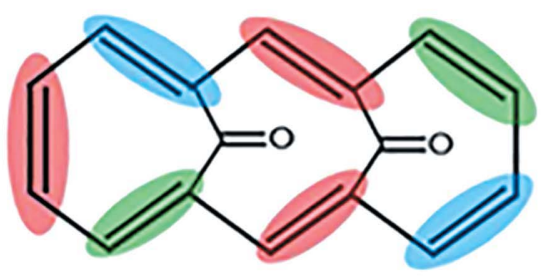

B

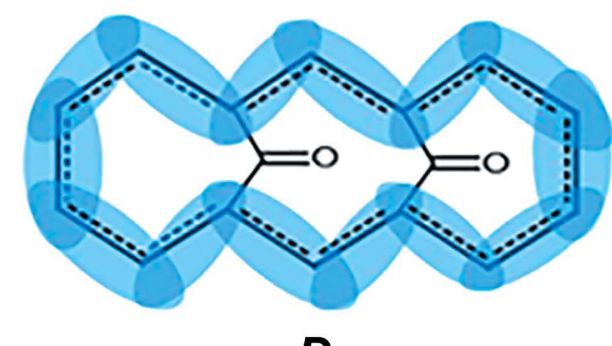

D

Figure 5

Schematic representation of the different localization schemes adopted for the unconstrained ELMO calculations. Localizations schemes $A$ and $B$ are associated with resonance structures $A$ and $B$ in Fig. 1, respectively, while $D$ represents the localization scheme with only one fragment for the $14 \pi$ electrons. 
Table 4

Differences (in $\mathrm{kJ} \mathrm{mol}^{-1}$ ) between the ELMO energies at high pressure ( $7.7 \mathrm{GPa})$ and at ambient pressure for the three different localization schemes taken into account; all the acronyms are described in the text.

\begin{tabular}{llll}
\hline & $6-31 \mathrm{G}$ & $6-31 \mathrm{G}(\mathrm{d}, \mathrm{p})$ & cc-pVDZ \\
\hline$\Delta E_{D}$ & 43.21 & 45.76 & 56.10 \\
$\Delta E_{A}$ & -38.69 & -36.63 & -24.24 \\
$\Delta E_{B}$ & 155.50 & 163.20 & 175.30 \\
\hline
\end{tabular}

Table 5

Differences in $\mathrm{kJ} \mathrm{mol}^{-1}$ ) between the ELMO energies obtained with localization schemes $A$ and $B$ both at ambient and high $(7.7 \mathrm{GPa})$ pressures; all the acronyms are described in the text.

\begin{tabular}{llll}
\hline & $6-31 \mathrm{G}$ & $6-31 \mathrm{G}(\mathrm{d}, \mathrm{p})$ & cc-pVDZ \\
\hline$\Delta E_{A-B}^{A P}$ & -3.77 & -4.31 & -4.31 \\
$\Delta E_{A-B}^{H P}$ & -197.95 & -204.15 & -203.86 \\
\hline
\end{tabular}

pressure, the two resonance structures depicted in Fig. 1 should not contribute equally to the description of the 14electron Hückel system of the biscarbonyl[14]annulene molecule. Therefore, a completely delocalized representation of the $14 \pi$-electron system of BCA is less and less adequate at higher pressures (in agreement with the result obtained adopting scheme $D$ as the localization pattern).

These observations are further confirmed by the differences, at ambient and high pressures, between the ELMO energies corresponding to localization schemes $A$ and $B$

$$
\Delta E_{\mathrm{A}-\mathrm{B}}^{X}=E_{\mathrm{ELMO}-\mathrm{A}}^{X}-E_{\mathrm{ELMO}-\mathrm{B}}^{X},
$$

where, in this case, $X$ stands for AP ('ambient pressure') or HP ('high pressure').

From Table 5, it is easy to observe that, while at ambient pressure the two resonance structures of BCA are approximately energetically equivalent (structure $A$ only slightly more favourable than structure $B$ ), at $7.7 \mathrm{GPa}$, the resonance structure corresponding to localization scheme $A$ becomes significantly more stable. This is further evidence of the fact that, when pressure increases, the contributions of the two resonance structures in the description of electronic structure of BCA are no longer equivalent and that a partial rupture of aromaticity probably occurs.

4.2.3. XC-ELMO-VB computations. In order to obtain confirmation of the trend observed for the unconstrained ELMO calculations and to estimate the weight of resonance structures $A$ and $B$ of BCA at ambient and high pressures, we then performed X-ray-constrained ELMO valence bond computations.

As already mentioned in $\$ 3.4 .3$, our XC-ELMO-VB wavefunctions have been written as a linear combination of two predetermined unconstrained ELMO single Slater determinants: one corresponding to resonance structure $A$ and another one corresponding to resonance structure $B$.

As was done for the ELMO calculations, the XC-ELMOVB computations were also performed considering the molecular geometries of BCA associated with the crystallographic structures determined at ambient and high
Table 6

Statistical agreements $\chi^{2}$ and Chirgwin-Coulson coefficients obtained through the unconstrained ELMO-VB calculations $(\lambda=0.0)$ at ambient and high pressures.

\begin{tabular}{llllllll}
\hline & \multicolumn{3}{c}{ Ambient pressure } & & \multicolumn{3}{c}{ High pressure } \\
\cline { 2 - 3 } Basis set & $\chi^{2}$ & $K_{A}$ & $K_{B}$ & & $\chi^{2}$ & $K_{A}$ & $K_{B}$ \\
\hline 6-31G & 5.88 & 0.546 & 0.454 & & 6.31 & 0.989 & 0.011 \\
6-31G(d,p) & 5.15 & 0.559 & 0.441 & & 5.64 & 0.992 & 0.008 \\
cc-pVDZ & 4.97 & 0.559 & 0.441 & & 5.68 & 0.992 & 0.008 \\
\hline
\end{tabular}

Table 7

Statistical agreements $\chi^{2}$ and Chirgwin-Coulson coefficients obtained through the X-ray-constrained ELMO-VB calculations at ambient and high pressures.

The value of the external multiplier $\lambda$ at convergence is also shown.

\begin{tabular}{|c|c|c|c|c|c|c|c|c|}
\hline \multirow[b]{2}{*}{ Basis set } & \multicolumn{4}{|c|}{ Ambient pressure } & \multicolumn{4}{|c|}{ High pressure } \\
\hline & $\lambda$ & $\chi^{2}$ & $K_{A}$ & $K_{B}$ & $\lambda$ & $\chi^{2}$ & $K_{A}$ & $K_{B}$ \\
\hline $6-31 \mathrm{G}$ & 0.05 & 5.88 & 0.504 & 0.496 & 0.77 & 6.18 & 0.593 & 0.407 \\
\hline $6-31 \mathrm{G}(\mathrm{d}, \mathrm{p})$ & 0.05 & 5.12 & 0.490 & 0.510 & 0.59 & 5.41 & 0.587 & 0.413 \\
\hline cc-pVDZ & 0.06 & 4.93 & 0.491 & 0.509 & 0.57 & 5.43 & 0.586 & 0.414 \\
\hline
\end{tabular}

(7.7 $\mathrm{GPa})$ pressures, and using the standard basis-sets $6-31 \mathrm{G}$, $6-31 G(d, p)$ and cc-pVDZ. Furthermore, for all the X-rayconstrained ELMO valence bond computations, we have used unit-cell parameters, ADPs and structure-factor amplitudes deposited with the corresponding crystal structures.

The results of the XC-ELMO-VB calculations at ambient and high pressures are shown in Tables 6 and 7. In Table 6, we have reported the values of the Chirgwin-Coulson weights associated with resonance structures $A$ and $B$, when the external parameter $\lambda$ is set equal to 0.0 (namely, unconstrained ELMO-VB computations). We can observe that for all the basis sets, at ambient pressure, resonance structure $A$ is slightly more important than resonance structure $B$. On the contrary, at high pressure, structure $A$ becomes largely predominant. Nevertheless, it is important to note that the unconstrained ELMO-VB calculations take into account only energetic aspects and practically confirm the results obtained by means of the simple unconstrained ELMO calculations discussed in $§ 4.2 .2$. In fact, the ELMO Slater determinants that were used to expand the unconstrained ELMO-VB wavefunctions were the ones resulting from the unconstrained ELMO calculations and they were kept frozen during the optimization of the coefficients $\left\{C_{j}\right\}$ in equation (15).

In Table 7, we have reported the results of the real XCELMO-VB computations. By exploiting experimental structure-factor amplitudes as external constraints, they allowed us to introduce electron-density-related aspects in the determination of the weights associated with resonance structures $A$ and $B$. In this case, for all the basis sets taken into account, we can note that while, at ambient pressure, structures $A$ and $B$ contribute equally to the description of the Hückel system (perfect aromaticity), at high pressure, resonance structure $A$ becomes slightly more important, thus partially breaking the aromaticity of the molecule. 
For the sake of completeness, in the supporting information we have also reported how the statistical agreement $\chi^{2}$ and the Chirgwin-Coulson coefficients vary in the function of the external parameter $\lambda$ for all the X-ray-constrained ELMOVB calculations that we have performed (see Figs. S2-S7 in the supporting information).

\section{Conclusions}

In this paper, we reported an accurate analysis of the electrondensity models calculated or refined for the BCA molecule under high pressure. This is the first systematic investigation of accurate charge density distribution from high-pressure X-ray diffraction data. It is interesting that the electron-density study confirms the hypothesis of a partial localization of one of the two resonant electronic configurations when the species is compressed. This was anticipated by the geometry refined from X-ray diffraction data and further confirmed by analysing: (i) the multipolar electron density, (ii) the computed X-ray-constrained canonical Hartree-Fock molecular orbitals, (iii) the results of the unconstrained ELMO calculations and, finally, (iv) the weights of the resonance structures provided by the newly developed XC-ELMO-VB method, a new theoretical method which is particularly adequate for this chemical problem.

Although very few accurate data sets have been collected at high pressure so far, one could envisage more data in the near future, thanks especially to the availability of synchrotron beamlines of very high-energy and dedicated set-ups for the experimental requirement of high-resolution data collection using diamond anvil cells. Of course, there is still room to improve the strategy of data collection and data quality. Nevertheless, it is obvious that the accuracy of these measurements cannot match the charge density data collection carried out at ambient pressure and low temperature. For this reason, it is important to warn that only significant changes of the charge distribution are presently detectable. These changes are, for example, those associated with different electronic states of molecules (or polymers) or different electronic configurations that imply a significant transfer of electron density from one bond (or one atom/ group) to another (as in BCA). On the other hand, it seems more difficult to detect smaller polarizations (in the absence of a configurational change) caused by the crystal internal electric field modified by pressure.

Having this in mind, we are currently planning experiments of species that, similarly to BCA, show severe rearrangements of the electron-density distribution upon compression. For these species, experimental measurements are essential because theoretical calculations are themselves challenged in these conditions and experimental validations are vital.

\section{Acknowledgements}

The authors thank Dr Heribert Whilelm and Dr Annette Kleppe for assistance during one of the experiments at Diamond Light Source, Professor Andrew Jephcoat for assistance during sample preparation and experiment planning, Professor Riccardo Destro and Dr Raffaella Soave for providing the crystal samples, and Dr Arianna Lanza for assistance in preparation of the drawings. The anonymous referees are thanked for their careful evaluation of this manuscript and for suggestions for the improvement. The authors declare no conflict of interest. This paper is dedicated to the memory of Professor P. Coppens. PM thanks Professor Coppens for many stimulating conversations and joint research over the past 20 years.

\section{Funding information}

The following funding is acknowledged: Swiss National Science Foundation (awards No. 160157 and 162861).

\section{References}

Abramov, Yu. A., Volkov, A. V. \& Coppens, P. (1999). Chem. Phys. Lett. 311, 81-86.

Adams, W. H. (1961). J. Chem. Phys. 34, 89-102.

Agilent (2014). CrysAlis PRO. Agilent Technologies Ltd, Yarnton, Oxfordshire, England.

Bader, R. F. W. (1990). Atoms in Molecules: A Quantum Theory. Oxford University Press.

Bader, R. F. W. \& Beddall, P. M. (1972). J. Chem. Phys. 56, 3320-3329.

Boldyreva, E. V. (2008). Acta Cryst. A64, 218-231.

Bürgi, H. B. \& Dunitz, J. (1994). Structure Correlation. Berlin: VCH Weinheim.

Bytheway, I., Grimwood, D. J., Figgis, B. N., Chandler, G. S. \& Jayatilaka, D. (2002). Acta Cryst. A58, 244-251.

Bytheway, I., Grimwood, D. J. \& Jayatilaka, D. (2002). Acta Cryst. A58, 232-243.

Casati, N., Kleppe, A., Jephcoat, A. \& Macchi, P. (2016). Nat. Commun. 7, 10901.

Casati, N., Macchi, P. \& Sironi, A. (2007). J. Appl. Cryst. 40, 628-630.

Chirgwin, B. H. \& Coulson, C. A. (1950). Proc. R. Soc. London Ser. A, 201, 196-209.

Clementi, E. \& Raimondi, D. L. (1963). J. Chem. Phys. 38, 2686-2689.

Clementi, E. \& Roetti, C. (1974). At. Data Nucl. Data Tables, 14, 177478.

Coppens, P. (1967). Science, 158, 1577-1579.

Coppens, P. (2005). Angew. Chem. Int. Ed. 44, 6810-6811.

Couty, M., Bayse, C. A. \& Hall, M. B. (1997). Theoret. Chim. Acta, 97, 96-109.

Destro, R. (1988). Aust. J. Phys. 41, 503-510.

Destro, R. \& Merati, F. (1995). Acta Cryst. B51, 559-570.

Deutsch, M., Gillon, B., Claiser, N., Gillet, J.-M., Lecomte, C. \& Souhassou, M. (2014). IUCrJ, 1, 194-199.

Dos Santos, L. H. R., Genoni, A. \& Macchi, P. (2014). Acta Cryst. A70, 532-551.

Elsässer, T. \& Woerner, M. (2012). Modern Charge-Density Analysis, edited by C. Gatti \& P. Macchi, pp. 695-712. Berlin: Springer.

Fabbiani, F. P. A., Dittrich, B., Pulham, C. R. \& Warren, J. E. (2011). Acta Cryst. A67, C376.

Fertey, P., Alle, P., Wenger, E., Dinkespiler, B., Cambon, O., Haines, J., Hustache, S., Medjoubi, K., Picca, F., Dawiec, A., Breugnon, P., Delpierre, P., Mazzoli, C. \& Lecomte, C. (2013). J. Appl. Cryst. 46, 1151-1161.

Fischer, A., Tiana, D., Scherer, W., Batke, K., Eickerling, G., Svendsen, H., Bindzus, N. \& Iversen, B. B. (2011). J. Phys. Chem. $A, \mathbf{1 1 5}, 13061-13071$.

Fornili, A., Sironi, M. \& Raimondi, M. (2003). J. Mol. Struct. Theochem, 632, 157-172.

Francisco, E., Pendás, A. M. \& Adams, W. H. (1992). J. Chem. Phys. 97, 6504-6508. 
Genoni, A. (2013a). J. Phys. Chem. Lett. 4, 1093-1099.

Genoni, A. (2013b). J. Chem. Theory Comput. 9, 3004-3019.

Genoni, A. (2016). Acta Cryst. A72, s163-s164.

Genoni, A. (2017). Acta Cryst. A73, 312-316.

Genoni, A., Dos Santos, L. H. R., Meyer, B. \& Macchi, P. (2017). IUCrJ, 4, 136-146.

Genoni, A. \& Meyer, B. (2016). Adv. Quantum Chem. 73, 333-362.

Gilbert, T. L. (1974). J. Chem. Phys. 60, 3835-3844.

Gillet, J.-M., Becker, P. J. \& Cortona, P. (2001). Phys. Rev. B, 63, 2351151-2351157.

Grimwood, D. J., Bytheway, I. \& Jayatilaka, D. (2003). J. Comput. Chem. 24, 470-483.

Grimwood, D. J. \& Jayatilaka, D. (2001). Acta Cryst. A57, 87-100.

Hansen, N. K. \& Coppens, P. (1978). Acta Cryst. A34, 909-921.

Hirshfeld, F. L. (1971). Acta Cryst. B27, 769-781.

Hirshfeld, F. L. (1976). Acta Cryst. A32, 239-244.

Huzinaga, S. \& Cantu, A. A. (1971). J. Chem. Phys. 55, 5543-5549.

Jayatilaka, D. (1998). Phys. Rev. Lett. 80, 798-801.

Jayatilaka, D. (2012). Modern Charge-Density Analysis, edited by C. Gatti \& P. Macchi, pp. 213-257. Dordrecht: Springer.

Jayatilaka, D. \& Grimwood, D. J. (2001). Acta Cryst. A57, 76-86.

Jayatilaka, D. \& Grimwood, D. J. (2003). Lect. Notes Comput. Sci. 2660, 142-151.

Katrusiak, A. (2008). Acta Cryst. A64, 135-148.

Lanza, A. (2016). PhD thesis, University of Bern, Switzerland.

Larsen, F. K. (1995). Acta Cryst. B51, 468-482.

Legrand, V., Carbonera, C., Pillet, S., Souhassou, M., Létard, F., Guionneau, P. \& Lecomte, C. (2005). J. Phys. Conf. Ser. 21, 73-80.

Macchi, P. (2013). Crystallogr. Rev. 19, 59-109.

Macchi, P., Bürgi, H.-B., Chimpri, A. S., Hauser, J. \& Gál, Z. (2011). J. Appl. Cryst. 44, 763-771.

Macchi, P. \& Casati, N. (2011). Acta Cryst. A67, C163-C164.

Madsen, A. Ø. (2006). J. Appl. Cryst. 39, 757-758.

Matsuoka, O. (1977). J. Chem. Phys. 66, 1245-1254.

McWeeny, R. (1959). Proc. R. Soc. London Ser. A, 253, 242-259.
McWeeny, R. (1960). Rev. Mod. Phys. 32, 335-369.

McWeeny, R. (1992). Methods of Molecular Quantum Mechanics. London: Academic Press.

Meindl, K. \& Henn, J. (2008). Acta Cryst. A64, 404-418.

Morgenroth, W., Overgaard, J., Clausen, H. F., Svendsen, H., Jørgensen, M. R. V., Larsen, F. K. \& Iversen, B. B. (2008). J. Appl. Cryst. 41, 846-853.

Ordejón, P., Drabold, D. A., Grumbach, M. P. \& Martin, R. M. (1993). Phys. Rev. B, 48, 14646-14649.

Pillet, S., Souhassou, M., Lecomte, C., Schwarz, K., Blaha, P., Rérat, M., Lichanot, A. \& Roversi, P. (2001). Acta Cryst. A57, 290-303.

Schmøkel, M. S., Bjerg, L., Overgaard, J., Larsen, F. K., Madsen, G. K. H., Sugimoto, K., Takata, M. \& Iversen, B. B. (2013). Angew. Chem. Int. Ed. Engl. 52, 1053-1056.

Smits, G. F. \& Altona, C. (1985). Theor. Cim. Acta, 67, 461-475.

Stevens, E. D. \& Coppens, P. (1976). Acta Cryst. A32, 915-917.

Stewart, R. F. (1970). J. Chem. Phys. 53, 205-213.

Stewart, R. F., Bentley, J. J. \& Goodman, B. (1975). J. Chem. Phys. 63, 3786-3793.

Stewart, R. F., Spackman, M. A. \& Flensburg, C. (2000). VALRAY User's Manual. Carnegie Mellon University, USA, and University of Copenhagen, Denmark.

Stoll, H., Wagenblast, G. \& Preuss, H. (1980). Theor. Chim. Acta, 57, 169-178.

Szekeres, Z. \& Surján, P. R. (2003). Chem. Phys. Lett. 369, 125130.

Tse, J. S., Klug, D. D., Patchkovskii, S., Ma, Y. \& Dewhurst, J. K. (2006). J. Phys. Chem. B, 110, 3721-3726.

Volkov, A., Macchi, P., Farrugia, L. J., Gatti, C., Mallinson, P., Richter, T. \& Koritsanszky, T. (2006). XD2006. University at Buffalo, State University of New York, NY, USA, University of Milano, Italy, University of Glasgow, UK, CNRISTM, Milano, Italy, Middle Tennessee State University, TN, USA.

Yamanaka, T., Okada, T. \& Nakamoto, Y. (2009). Phys. Rev. B, 80, 094108. 